\title{
Non-standard pairing in asymmetric trapped Fermi gases
}

\author{
Paolo Castorina, ${ }^{1}$ Marcella Grasso, ${ }^{1,2,3}$ Micaela Oertel, ${ }^{4}$ Michael Urban, ${ }^{3}$ and Dario Zappalà ${ }^{2}$ \\ ${ }^{1}$ Dipartimento di Fisica e Astronomia, Via Santa Sofia 64, I-95123 Catania, Italy \\ ${ }^{2}$ INFN, Sezione di Catania, Via Santa Sofia 64, I-95123 Catania, Italy \\ ${ }^{3}$ Institut de Physique Nucléaire, 15 rue Georges Clémenceau, F-91406 Orsay Cedex, France \\ ${ }^{4}$ Service de Physique Nucléaire, CEA-DAM Ile-de-France, BP 12, F-91680 Bruyères-le-Châtel, France
}

\begin{abstract}
We study an ultracold trapped Fermi gas of atoms in two hyperfine states with unequal populations. In this situation the usual BCS pairing is suppressed and non-standard pairing mechanisms become important. These are treated by solving the Bogoliubov-de Gennes equations, which at the same time correctly take into account the finite size of the trapped system. We find results which can be viewed as generalization of the LOFF phase to finite systems.

PACS numbers: 03.75.Ss,21.60.Jz
\end{abstract}

In the last years much progress has been made in improving the techniques used to trap and cool dilute gases of bosonic and fermionic atoms [1, 2]. One of the interesting aspects of the properties of ultra-cold gases is that the interatomic interaction can be modified, both in its intensity and in its sign, by changing the applied magnetic field around a Feshbach resonance. Due to the very low densities and temperatures in these systems the details of the interatomic interaction are unimportant and the interaction can be characterized by one single parameter, the $s$-wave scattering length $a$. In this article we consider Fermi gases trapped and cooled in two hyperfine states with an attractive interaction, i.e., $a<0$. We will concentrate on the weakly interacting case $\left(k_{F}|a| \ll 1\right.$, where $k_{F}$ denotes the Fermi momentum). In this region, BCS superfluidity with formation of Cooper pairs is expected below a certain critical temperature. So far, some experimental signals have been found which would indicate the existence of superfluidity in this region [2], but a clear evidence is still missing.

Besides the interaction, also the population of the two hyperfine states can experimentally be controlled. Usually mixtures with equal populations are created, but controlled asymmetric mixtures have also been obtained [3]. Unequal populations of the two hyperfine states can lead to very interesting phenomena. For instance, the BCS pairing mechanism is supposed to become suppressed [4] since the two Fermi momenta associated with the two spin polarizations become different: The formation of zero-momentum Cooper pairs built of two atoms at their respective Fermi surface becomes difficult. Instead, other more exotic pairing phenomena have been suggested for the case of unequal populations, like the Larkin-Ovchinnikov-Fulde-Ferrel (LOFF) phase [5], the Sarma (interior gap) phase 6, 7], or a phase with deformed Fermi surfaces (DFS) 8]. Many of these non-standard pairing mechanisms have already been discussed in other domains of physics where asymmetric two-component fermion systems can be found: Superconductors in a magnetic field [9, 10], neutron-proton pairing in asymmetric nuclear matter 11], color superconductivity in high density QCD [12]. The experimental observation of the LOFF phase in the case of super- conductivity is a subject of debate. It seems that quite recently an oscillating superconducting order parameter has been observed in a ferromagnetic thin film [13].

Usually [4, 5, 6, 6, 8] these non-standard pairing types in ultracold Fermi gases are usually discussed for the case of infinite and homogeneous systems, or for trapped systems in local-density approximation (LDA) which again amounts to treating the system locally as homogeneous. However, as we are going to show, in certain cases the LDA can become a very poor approximation and we therefore want to analyze this problem within a fully microscopic mean field Bogoliubov-de Gennes (BdG) approach 14 taking into account the inhomogeneity and finite size of the trapped system. Recently the solution of the BdG equations has been considered in Ref. 15], where the authors discuss also possibilities for the experimental detection of a spatially modulated pairing gap.

In the present article we study two systems: a small one where shell effects still play a role and a relatively large one, where one could expect the LDA to be a reasonable approximation. As we will show, this is not always the case, although the LDA describes roughly the qualitative features. In addition, we examine the temperature dependence of the non-standard pairing phase, since this is important in connection with the experimental realization of such a phase.

Let us briefly recall the BdG formalism. We consider a system containing $N=N_{+}+N_{-}$atoms of mass $m$ trapped by a spherical harmonic potential in two hyperfine states denoted by + and - . The many-body Hamiltonian of the system can be written as

$$
\begin{aligned}
H=\sum_{\sigma} \int d^{3} r( & \Psi_{\sigma}^{\dagger}(\mathbf{r}) H_{0} \Psi_{\sigma}(\mathbf{r}) \\
& \left.+g \Psi_{+}^{\dagger}(\mathbf{r}) \Psi_{-}^{\dagger}(\mathbf{r}) \Psi_{-}(\mathbf{r}) \Psi_{+}(\mathbf{r})\right)
\end{aligned}
$$

where $H_{0}=-\hbar^{2} \nabla^{2} /(2 m)+m \omega^{2} r^{2} / 2$ denotes the harmonic oscillator single-particle Hamiltonian, $\Psi_{\sigma}(\mathbf{r})$ is the field operator which annihilates a particle at the position $\mathbf{r}$ in the spin state $\sigma(+$ or -$), g=4 \pi \hbar^{2} a / m$ is the coupling constant. In mean-field approximation, on can derive the following $\mathrm{BdG}$ equations corresponding to the 
Hamiltonian (11):

$$
\begin{aligned}
u_{\eta \sigma}(\mathbf{r}) E_{\eta \sigma} & =W_{\sigma} u_{\eta \sigma}(\mathbf{r})+\Delta(\mathbf{r}) v_{\eta-\sigma}(\mathbf{r}), \\
v_{\eta-\sigma}(\mathbf{r}) E_{\eta \sigma} & =-W_{-\sigma} v_{\eta-\sigma}(\mathbf{r})+\Delta(\mathbf{r}) u_{\eta \sigma}(\mathbf{r}),
\end{aligned}
$$

where $W_{\sigma}=H_{0}+g \rho_{-\sigma}(\mathbf{r})-\mu_{\sigma}, \mu_{\sigma}$ and $\rho_{\sigma}$ being the chemical potential and the density, respectively. $\Delta(\mathbf{r})$ denotes the pairing field (gap) and $E_{\eta \sigma}, u_{\eta \sigma}$ and $v_{\eta \sigma}$ are the quasiparticle energy and wave functions, respectively, corresponding to the quantum numbers $\eta=n, l, m$ and spin $\sigma$. In order to have different populations, the two chemical potentials $\mu_{+}$and $\mu_{-}$must be different. It is convenient to introduce the average chemical potential $\bar{\mu}$ and to write $\mu_{\sigma}=\bar{\mu}+\sigma \delta \mu / 2$ where $\delta \mu$ determines the asymmetry. Eqs. (2) reduce to the usual BdG equations in the symmetric case $\mu_{+}=\mu_{-}$. They are solved numerically employing the same regularization method for the pairing field as described in Ref. 16] for the symmetric case.

Eqs. (2) are general enough to describe rather complicated types of non-standard pairing. In the case of usual BCS pairing, the dominant matrix elements of the pairing field are the diagonal ones, i.e., each state $|n, l, m,+\rangle$ is paired with its time-reversed counterpart $|n, l,-m,-\rangle$. However, the non-diagonal matrix elements of $\Delta$ are also included, which amounts to taking into account also the pairing of states $|n, l, m,+\rangle$ and $\left|n^{\prime}, l,-m,-\right\rangle$ with different principal quantum numbers $n^{\prime} \neq n$. In our present calculation, we still keep the restriction that the Cooper pairs have total angular momentum zero. To release this constraint would mean to allow for a spontaneous breakdown of spherical symmetry, which would be numerically very heavy. The effect of strong non-diagonal matrix elements of $\Delta$ in fact corresponds closely to the LOFF phase in the case of a uniform system. There, the states are labeled by their momentum $\mathbf{k}$. In the simplest version of the LOFF phase, the Cooper pairs have total momentum q, i.e., each state $|\mathbf{k},+\rangle$ is paired with $|-\mathbf{k}+\mathbf{q},-\rangle$. The corresponding gap is oscillating with wave vector $\mathbf{q}$ and its matrix elements are therefore of the form $\Delta_{\mathbf{k k}^{\prime}}=\Delta \delta_{\mathbf{k}-\mathbf{a} \cdot \mathbf{k}^{\prime}}$ (in contrast to the BCS phase, where $\left.\Delta_{\mathbf{k k}^{\prime}}=\Delta \delta_{\mathbf{k k}^{\prime}}\right)$ [17.

In the discussion of our results all quantities will be given in harmonic oscillator units. We use the same coupling constant as in Ref. [16], i.e., $g=-\hbar^{2} l_{h o} / m$, where $l_{h o}=\sqrt{\hbar /(m \omega)}$ denotes the harmonic oscillator length, and we consider two values for the average chemical potential, $\bar{\mu}=22 \hbar \omega$ (small system with $N \approx 4900$ ) and 32 $\hbar \omega$ (large system with $N \approx 17000$ ).

In Fig. 1 we show the values of the pairing gap $\Delta(0)$ at the center of the trap for increasing aymmetry $\delta \mu$ at $T=0$. Let us first look at the lowest line, corresponding to the small system with $\bar{\mu}=22 \hbar \omega$. When both spin states are equally populated $(\delta \mu=0)$, we find $\Delta(0) \approx 2$ $\hbar \omega$, i.e., we are no more in the intrashell-pairing regime, but shell effects are still important [16]. If we increase $\delta \mu$, the two Fermi surfaces become more and more separated, i.e., if the state $|n, l, m,+\rangle$ lies close to the Fermi level for

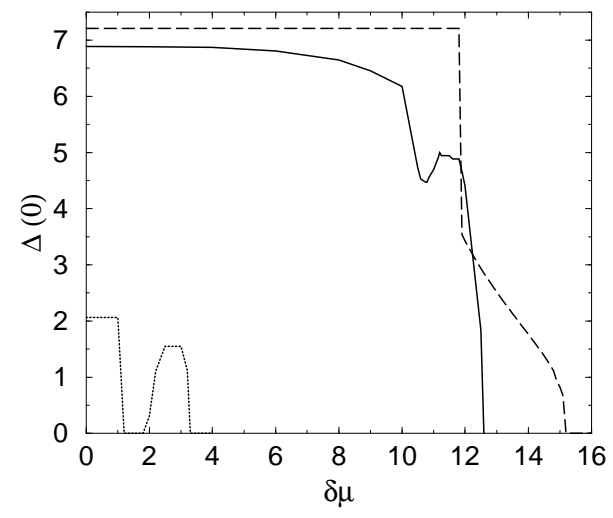

FIG. 1: Value of the pairing gap at the center of the trap (in units of $\hbar \omega$ ) as a function of the asymmetry $\delta \mu$ (in units of $\hbar \omega)$. The lowest line corresponds to $\bar{\mu}=22 \hbar \omega$. The two upper lines correspond to $\bar{\mu}=32 \hbar \omega$ and show the BdG (solid line) and the LDA result (dashed line).

spin + , the state $|n, l,-m,-\rangle$ lies far from the Fermi level for spin -, making BCS pairing less and less favorable. As a consequence, at $\delta \mu \approx 1.2 \hbar \omega$, corresponding to a particle number asymmetry $\alpha=\left(N_{+}-N_{-}\right) / N \approx 0.06$, the pairing disappears (shell closure effect). But then, near $\delta \mu \approx 2 \hbar \omega(\alpha \approx 0.07)$, the states $|n, l, m,+\rangle$ near the Fermi level for spin + approach the states $\left|n^{\prime}, l,-m,-\right\rangle$ near the Fermi level for spin - if $n^{\prime}=n-1$. Therefore, pairing becomes again possible, but now the Cooper pairs are built of two wavefunctions with different numbers of nodes, leading to a gap $\Delta(r)$ which as a function of $r$ has exactly one node.

Let us now turn to the investigation of the larger system, $\bar{\mu}=32 \hbar \omega$. Here it seems to be appealing to estimate if and where the LOFF phase could appear by using the LDA, which should be exact in an infinite system. In order to do this, we calculate at each point $R$ the thermodynamic potential $\Omega$ of a uniform gas with effective average chemical potential $\bar{\mu}_{\text {eff }}(R)=\bar{\mu}-m \omega^{2} R^{2} / 2$, assuming a gap of the form $\Delta(\mathbf{r})=\Delta \exp (i \mathbf{q} \cdot \mathbf{r})$, and minimize $\Omega$ with respect to $\Delta$ and $q$. To be more precise, we should have taken a more sophisticated expression for the order parameter, but we stress here that we use the LDA just to have some indications about what kind of behavior one should expect. Let us again look at $\Delta(0)$ as a function of the asymmetry (dashed line in Fig. 1). We find that LDA gives the BCS solution $q=0$ as the most favorable solution up to $\delta \mu=11.9 \hbar \omega$. At that asymmetry we find a first-order phase transition (i.e., a discontinuity in $\Delta(0))$ to the LOFF phase with $q \sim l_{h o}^{-1}$ which means that the order parameter oscillates with a wavelength of $\sim 6.2 l_{h o}$. This behavior is different from the microscopic $(\mathrm{BdG})$ result (solid line in Fig. 1), which shows a smooth behavior of $\Delta(0)$. Nevertheless, also in the BdG calculation there is a rapid change of $\Delta(0)$ between $\delta \mu=10 \hbar \omega(\alpha \approx 0.25)$ and $\delta \mu=11 \hbar \omega(\alpha \approx 0.29)$, where the system goes from the BCS-type to the LOFF- 


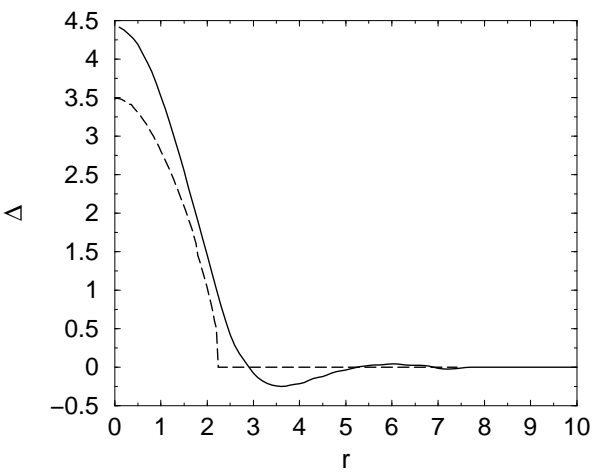

FIG. 2: Radial profile of the pairing gap $\Delta(r)$ (in units of $\hbar \omega$ ) for $\delta \mu=12 \hbar \omega$. The radial coordinate $r$ is expressed in units of $l_{h o}$. The microscopic (solid line) and the LDA (dashed line) results are plotted.

type phase, as discussed above. The minimum that one observes for the BdG gap at $\delta \mu \approx 10.8 \hbar \omega$ and the subsequent enhancement are due to shell effects which still persist even in this large system and which cannot be reproduced by the semiclassical LDA calculation.

In Fig. 2 we plot the radial profile of the pairing field $\Delta(r)$ for $\delta \mu=12 \hbar \omega$, corresponding to $\alpha \approx 0.3$, at $T=0$. The microscopic (solid line) and the LDA (dashed line) results are shown. Within LDA, in this case, the LOFF phase is more favorable than BCS for all values of $r$. We observe in Fig. 2 that the LDA gap goes abruptly to zero at a radius of $\sim 2 l_{h o}$, which is smaller than the LDA wavelength of $\sim 6.2 l_{h o}$. Thus, the region where the gap is non-zero does not even contain one wavelength of the oscillation and therefore the validity of LDA seems to be very questionable. As expected from the symmetric case [16], the LDA fails to describe the tail of the pairing field: The LDA gap goes abruptly to zero while in the microscopic case the gap has a smooth profile. We finally observe that the microscopic order parameter makes an oscillation and that a node is situated at $\approx 3 l_{h o}$ : The modulation of the order parameter and the presence of a node are signals which indicate that the system is in a LOFF-type phase.

Let us consider now the case of a smaller asymmetry, $\delta \mu=6 \hbar \omega$, corresponding to $\alpha \approx 0.15$. We show in Fig. 3 the radial profiles of the gap $\Delta(r)$ (top) and of the densities (bottom) at $T=0$. In the upper panel we report the microscopic (BdG) gap (solid line) and the LDA result (dashed line). In this case, according to the LDA, the BCS phase $(q=0)$ would be energetically preferred in the center of the gas (as we have shown in Fig. 1) and up to $r=3.8 l_{h o}$, while the LOFF phase with $q \sim 0.7 l_{h o}^{-1}$ would be more favorable in the interval $3.8 l_{h o}<r<4.1 l_{h o}$. For larger values of $r$, the LDA predicts that the gap should be zero. The wavelength of the oscillation of the order parameter in the LOFF phase $\left(\sim 8.9 l_{h o}\right)$ would again be much larger than the region where the gap is non zero. Contrary to the LDA,

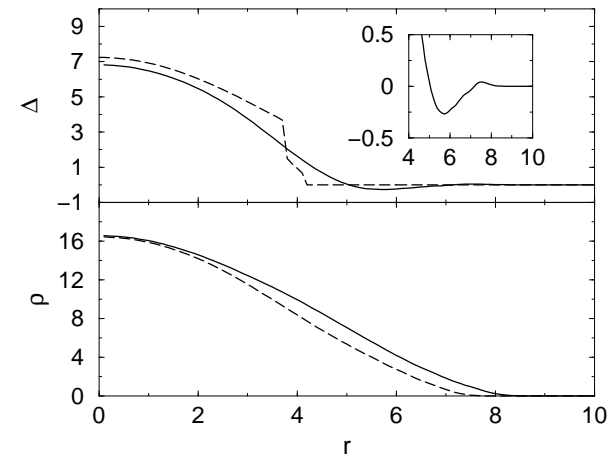

FIG. 3: Radial profiles of the pairing gap $\Delta(r)$ in units of $\hbar \omega$ (top) and of the densities in units of $l_{h o}^{-3}$ (bottom) for $\delta \mu=6$ $\hbar \omega$ as a function of the radial coordinate $r$ in units of $l_{h o}$. In the upper panel the solid and dashed lines correspond to the $\mathrm{BdG}$ and LDA results, respectively. In the lower panel, the solid and the dashed lines refer to the BdG results for the + and - densities, respectively.

the microscopic BdG calculation gives a smooth behavior of the order parameter. Near the center, it corresponds rather well to the LDA prediction, indicating that the pairing is more or less of BCS type. Between $r=4$ $l_{h o}$ and $10 l_{h o}$, the gap is oscillating (see inset in the upper panel of Fig. 3), indicating the appearance of the LOFF-type phase. Although within the BdG calculation there is no sharp transition from one phase to the other, qualitatively it seems that both phases can be present at the same time in different regions of the system.

In the lower panel of Fig. 3 the BdG results for the two densities $\left(\rho_{+}\right.$and $\left.\rho_{-}\right)$are shown. One observes that in the center of the gas the two densities are equal. This is coherent with the fact that in the BCS phase at $T=0$ the LDA always gives $\rho_{+}=\rho_{-}$if $\Delta>\delta \mu / 2$, as it is the case here.

All the results shown so far refer to $T=0$. However, in real experiments with trapped atomic gases the temperature is always non-zero. Let us therefore raise the temperature in the case of asymmetry $\delta \mu=6 \hbar \omega$ in order to analyze what happens to the gap modulation when the temperature is finite. In Fig. 4 we show the order parameter in the radial interval from $4 l_{h o}$ to $10 l_{h o}$ (where we observed an oscillation in the case $T=0$ ) for four values of temperature, $T=0$ (solid line), $T=0.5 \hbar \omega / k_{B}$ (dashed line) $T=\hbar \omega / k_{B}$ (triangles) and $T=2 \hbar \omega / k_{B}$ (circles). One observes that the oscillation has a smaller and smaller amplitude with increasing temperature and that it disappears between $T=\hbar \omega / k_{B}$ and $T=2 \hbar \omega / k_{B}$. Our interpretation of this result is that the critical temperature of the LOFF-type phase is smaller than the BCS critical temperature. Therefore, the LOFF-type phase disappears at some temperature between $\hbar \omega / k_{B}$ and 2 $\hbar \omega / k_{B}$, while the BCS gap in the central region of the gas is still different from zero at $T=2 \hbar \omega / k_{B}$. In this case the LDA results (not shown) are very different from the BdG ones (the gap is much too large), as one could ex- 


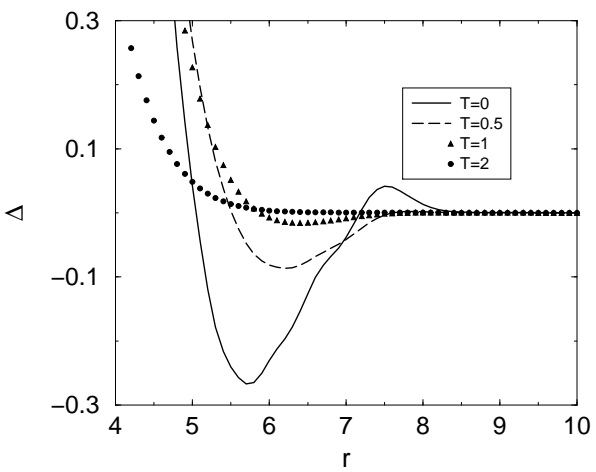

FIG. 4: Radial profile of the pairing gap $\Delta(r)$ (in units of $\hbar \omega$ ) for $\delta \mu=6 \hbar \omega$. The radial coordinate $r$ is expressed in units of $l_{h o}$. Results at $T=0$ (solid line), $T=0.5 \hbar \omega / k_{B}$ (dashed line) $T=\hbar \omega / k_{B}$ (triangles) and $T=2 \hbar \omega / k_{B}$ (circles) are reported.

pect from the fact that already in the symmetric case the agreement between LDA and BdG calculations becomes quite poor at finite temperature [16]. Nevertheless, qualitatively the LDA gives again the right indication: Also within LDA the LOFF phase is absent at $T=2 \hbar \omega / k_{B}$. Instead, at that temperature the Sarma phase becomes more favorable in certain regions within the LDA: This phase is characterized by zero momentum Cooper pairs, a gap $\Delta$ smaller than $\delta \mu / 2$, different densities $\rho_{+}$and $\rho_{-}$ and typical occupation number distributions as shown in Ref. 6].

To summarize, we have solved the BdG equations for an atomic Fermi gas with different populations of two hy- perfine states. It is well-known that an increasing asymmetry of the populations renders BCS pairing difficult, and non-standard pairing mechanisms become possible. In this article we showed that the BdG formalism automatically includes such non-standard pairing mechanisms through the non-diagonal matrix elements of the gap. For example, in the case of a small system, we found that the usual pairing disappears at a certain asymmetry, but when the asymmetry is strong enough such that the single-particle energies of states with opposite spin and different principal quantum numbers start to match, pairing becomes again possible, but now with an oscillating order parameter. This is very similar to the LOFF phase introduced for the case of a homogeneous system. In the case of a larger system, there is no longer a sharp separation between the BCS pairing and the LOFF-type pairing: As a function of asymmetry, but also as a function of the distance from the center of the trap, the system undergoes smooth transitions from one kind of pairing to the other. This result is qualitatively different from that obtained with LDA calculations, where the transition between the BCS and the LOFF phase is a first order phase transition. We also observe that even a system containing 17000 atoms is still much too small for the LDA to be applicable, since the wavelength of the LOFF oscillations is of the same order of magnitude as the whole system. Finally we looked at the temperature dependence of the LOFF-type phase. We observe that it disappears already at temperatures where the BCS phase is still present. This, of course, can be a problem if one tries to observe the LOFF phase in experiments.

We acknowledge discussions with M. Baldo, F. Cataliotti and A. Sedrakian.
[1] B. De Marco and D.S. Jin, Science 285, 285 (1999); M. Greiner, C.A. Regal, D.S. Jin, Nature 426, 537 (2003); M.W. Zwierlein, et al., Phys. Rev. Lett.91, 250401 (2003); C.A. Regal, M. Greiner, and D.S. Jin, Phys. Rev. Lett. 92, 040403 (2004); M.W. Zwierlein, et al., Phys. Rev. Lett. 92, 120403 (2004); M. Bartenstein, et al., Phys. Rev. Lett. 92, 120401 (2004); T. Bourdel, et al., Phys. Rev. Lett. 93, 050401 (2004).

[2] J. Kinast, S.L. Hemmer, M.E. Gehm, A. Turlapov, and J.E. Thomas, Phys. Rev. Lett. 92, 150402 (2004); C. Chin, et al., Science 305, 1128 (2004).

[3] M.W. Zwierlein, et al., Phys. Rev. Lett. 91, 250404 (2003).

[4] M. Houbiers et al., Phys. Rev. A 56, 4864 (1997).

[5] R. Combescot and C. Mora, Europhys. Lett. 68, 79 (2004).

[6] W.V. Liu and F. Wilczek, Phys. Rev. Lett. 90, 047002 (2003).

[7] P.F. Bedaque, H. Caldas, and G. Rupak, Phys. Rev. Lett. 91, 247002 (2003); J. Carlson and Sanjay Reddy, cond-mat/0503256

[8] A. Sedrakian et al., cond-mat/0404577

[9] P. Fulde and R.A. Ferrel, Phys. Rev.135, A550 (1964); A.I. Larkin and Yu.N. Ovchinnikov, Sov. Phys. JETP 20,
$762(1965)$.

[10] G. Sarma, Phys. Chem. Solids 24, 1029 (1963).

[11] A. Sedrakian and U. Lombardo, Phys. Rev. Lett. 84, 602 (2000).

[12] J.A. Bowers and K. Rajagopal, Phys. Rev. D 66, 065002 (2002); I. Shovkovy and M. Huang, Phys. Lett. B 564, 205 (2003); R. Casalbuoni and G. Nardulli, Rev. Mod. Phys. 76, 263 (2004).

[13] T. Kontos, M. Aprili, J. Lesueur, and X. Grison, Phys. Rev. Lett. 86, 304 (2001).

[14] P.-G. de Gennes, Superconductivity of Metals and Alloys (Benjamin, New York, 1966).

[15] T. Mizushima, K. Machida, and M. Ichioka, Phys. Rev. Lett. 94, 060404 (2005).

[16] M. Grasso and M. Urban, Phys. Rev. A 68, 033610 (2003).

[17] Note, however, that this analogy between the trapped and the homogeneous system is not perfect, since in the trapped system with $\delta \mu \neq 0$ even pairing between states $|n, l, m,+\rangle$ and $|n, l,-m,-\rangle$ includes pairing of states with different wave functions due to the different mean fields felt by atoms with different spin projections. 